\title{
Clustering of adenomas in the large intestine
}

\author{
T J EIDE AND T SCHWEDER
}

\begin{abstract}
From the Institute of Medical Biology and Institute of Mathematical and Physical Sciences, University of Troms $\varnothing$, Troms $\varnothing$, Norway
\end{abstract}

SUMMARY In a necropsy study of 68 men and 36 women with single or multiple adenomas of the large intestine the distribution of all adenomas within the bowel in individual patients was examined. Each adenoma was recorded both by its distance in centimetres from the caecal pole and by the intestinal segment in which it was found. Adenomas in individual patients were shown to occur significantly closer together than expected from the general distribution of adenomas within each age group. The statistically significant, but not very strong effect of clustering may be caused by the existence of one or more fields of the large intestine specific to individuals who have a higher than average propensity for neoplastic development.

In a previous study in a population at intermediate risk of colorectal cancer, we have shown that adenomas are most frequent in the distal half of the large intestine at ages below 65 years, and most frequent in the proximal half at older ages. Thus, an age related site specificity of adenomas in the large intestine has been shown on a population basis. ${ }^{1} \mathrm{We}$ know of no work, however, which examines whether adenomas tend to cluster in the large intestine in the individual patient or not.

The present study is a further analysis of the same material to investigate the distribution of adenomas in the large intestine in each patient. Do they show the degree of scatter expected from the general distribution of adenomas or is there a higher degree of clustering in the individual patient? By 'clustering' we mean that some of the multiple adenomas tend to occur closer together in the large intestine in individual patients than could be expected from the general distribution of adenomas. Any group of two or more adenomas will be understood as clustering and clustering may occur in more than one field within the large intestine in each individual.

\section{Methods}

MATERIAL

During a period of 22 months from 1974-76 the large intestines from consecutive necropsies were examined for the presence of polyps. Not included

Address for correspondence: T J Eide, Institute of Medical Biology, University of Troms $\emptyset$, Troms $\emptyset$, Norway.

Received for publication 27 January 1984 in the study were patients under 20 years of age, patients in which more than 72 hours had passed from the time of death to necropsy and patients who had undergone surgical resection of the large intestine.

With the large intestine in situ, the right and left flexures, the proximal end of the sigmoid colon and the rectosigmoid junction were marked with safety pins. After removal, the specimen was opened, washed, pinned out on a cork plate, and immersed in $4 \%$ neutral buffered formalin. The mucosa was examined under an illuminating magnifying lens $(2 \times)$, and all pedunculated or sessile lesions that measured $1 \mathrm{~mm}$ or more at their largest diameter were excised with adjacent normal mucosa for histological examination. The position of each polypoid lesion in the large intestine was recorded both by intestinal segment, and by its distance in centimetres from the caecal pole. The polyps were classified according to the WHO classification. ${ }^{2}$

\section{Results}

\section{ANALYSIS}

A total of 280 adenomas were found in 68 of 171 men and in 36 of 109 women. The ages of the youngest patients with one or more adenomas were 34 and 40 years and those of the oldest 90 and 93 years, in men and women respectively. Of the 280 patients examined, three colorectal carcinomas were found, of which two were in a 40 year old woman with 18 adenomas, which was the maximum number of adenomas in one patient.

In Figure 1 each adenoma in each patient having 
Fig. 1 Relative position of each adenoma between the caecal pole (0) and the lower end of rectum (1) in each individual in both men and women before and after the age of 65. Each line represents the large intestine of one patient. The lines are arranged according to increasing age of the patients from top to bottom within each sex and age group.
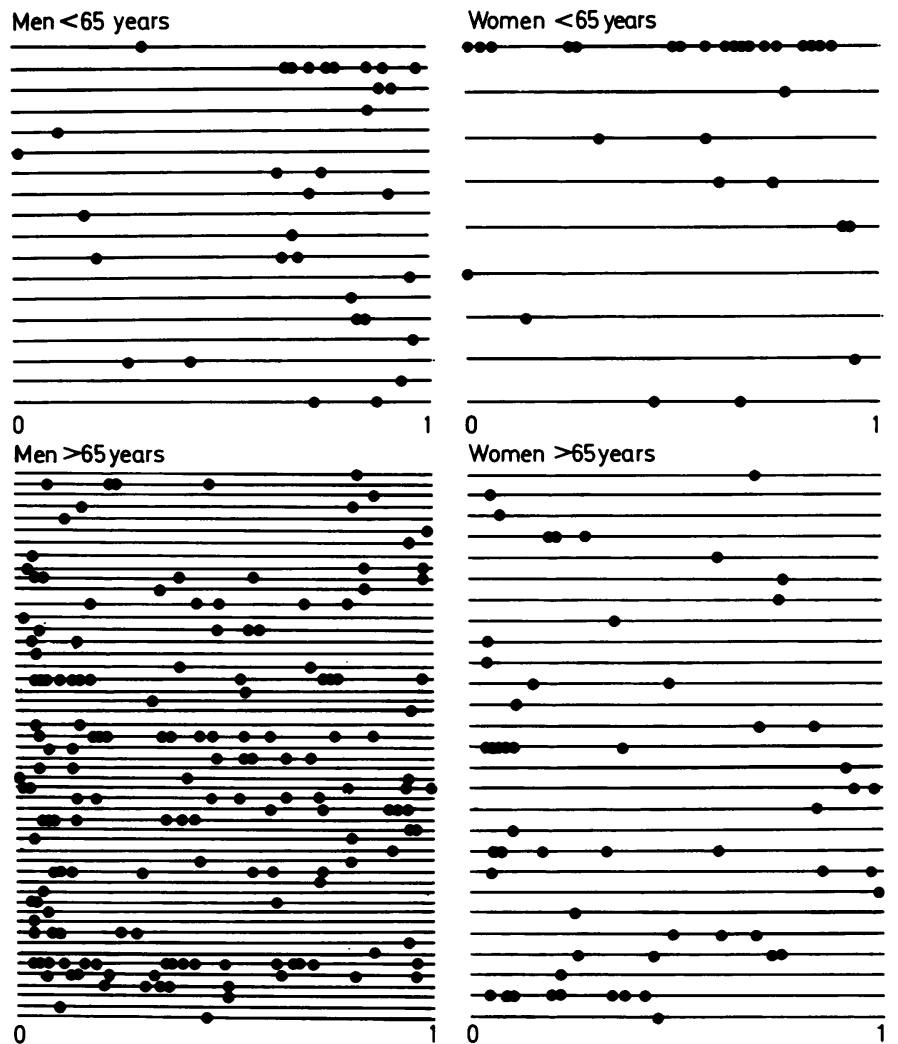

single or multiple adenomas is plotted on a line relative to the caecal pole and distal end of the rectum which shows the position of each adenoma as the length from the caecal pole relative to the total length of the large intestine. For all the four age and sex groups there appears to be a tendency towards individual clustering. The effect of clustering seems to be more marked in some patients than in others.

The question to be asked is whether the adenomas in the large intestine within individuals show significant clustering compared with the distribution of all adenomas in each age and sex group. To test the hypothesis of no difference in distribution of adenomas between individuals within age and sex groups, an analysis of variance was carried out. Because of the uneven distribution of adenomas in the large intestine, however, we used the nonparametric variance analysis by Kruskal and Wallis. ${ }^{3}$ Each adenoma in the respective sex and age group (cases with single adenomas were also included) was ranked according to the relative position between the caecal pole and the lower end of the rectum in the pooled material. Heterogeneity in placement distribution was then measured by the variance in the rank sums of adenomas between individuals.

The Kruskal and Wallis method is a reasonably sensitive method if individuals tend to have only one cluster of adenomas each, which is shifted in location between individuals. But as shown in Figure 1 there are also many patients with multiple clusters. To our knowledge there is no statistical method appropriate for testing the degree of clustering in patients with more than one cluster and therefore an alternative test to the Kruskal and Wallis method was designed. To adjust for the unevenness in the placement distribution of adenomas, this test is also based on the ranks of the adenomas. The idea is that under clustering there will be many relatively small distances between neighbouring adenomas of individuals. Heterogeneity between individuals may then be measured by a test based on the differences in the ranks of neighbouring adenomas within individuals. The method is described in detail elsewhere. ${ }^{4}$

From the Table it is seen that both of the two rank tests give evidence for clustering. It is not surprising 
Table Analysis of individual clustering of adenomas of the large intestine based on the non-parametric variance analysis of Kruskal and Wallis $(K W)$ and a test statistic based on the differences in the ranks of neighbouring adenomas within individuals $(S)$

\begin{tabular}{cllllc}
\hline $\begin{array}{l}\text { Sex and } \\
\text { age group }\end{array}$ & $\begin{array}{l}\text { Indivi- } \\
\text { duals } \\
(\text { no) }\end{array}$ & $\begin{array}{l}\text { Adenomas } \\
\text { (no) }\end{array}$ & $\begin{array}{l}\text { Statis- } \\
\text { tical } \\
\text { method }\end{array}$ & $\begin{array}{l}\text { Observed } \\
\text { test }\end{array}$ & $\begin{array}{l}\text { values } \\
\text { Estimated } \\
\text { p values* }\end{array}$ \\
\hline Men & & & KW & 65.8 & $2 \%$ \\
$65+$ & 50 & 162 & S & 43.9 & $<1 \%$ \\
Women & & & KW & 39.8 & $<1 \%$ \\
$65+$ & 27 & 55 & S & 12.9 & $<1 \%$ \\
Men & & & KW & 25.1 & $1 \%$ \\
$20-64$ & 18 & 33 & S & 5.7 & $3 \%$ \\
Women & & & KW & 13.0 & $5 \%$ \\
$20-64$ & 9 & 30 & S & 5.5 & $5 \%$ \\
\hline
\end{tabular}

* The $\mathrm{p}$ values were determined by estimating the null distribution of the test by, for each sex and age group, repeating 100 times a pseudo experiment by randomly allocating the adenomas to the various individuals from the pooled set of adenomas. This was done as a Monte Carlo experiment on a computer.

that the rank distance method (S) shows this most convincingly as this method presumably is more sensitive in the case of multiple clustering. In men less than 65 years of age the Kruskal and Wallis method shows the highest degree of significance as most patients having multiple adenomas in this group had two adenomas (only one cluster).

In addition to the above two tests we obtained the cumulative distribution function of distances between two adenomas when the adenomas were taken within individuals $\left(F_{w}\right)$ and from the pooled set of adenomas $\left(\mathrm{F}_{\mathrm{p}}\right)$. Clustering produces relatively more pairs of adenomas close together when the pairs are taken within individuals. As seen in Figure $2, F_{w}$ as a function of distance rises more sharply than $F_{p}$ in all sex and age groups but most marked in women above and in men below 65 years. Except for young women, $F_{w}$ moves to or outside its $95 \%$ tolerance limit, indicating significant clustering at the $5 \%$ level.

As we already know that there is a tendency for older people to have their adenomas in the proximal part of the large intestine and younger individuals their adenomas in the distal part, we have to rule out the possibility that individual clustering within our broad age groups is nothing but an age effect. Older women and younger males most significantly showed the effect of clustering in the respective age groups. These two groups were therefore examined in more details concerning a possible influence of age on the shown effect of clustering. By dividing the individuals in five year age groups and by looking at interadenoma distances within age groups, the within age group distribution is almost entirely within its tolerance limit (dashed lines) as shown in Figure 3. This indicates that age does not significantly contribute to the shown effect of individual clustering.

We were also interested in how clustering of adenomas was related to the anatomical segments of the large intestine. For each of the segments, now called the index segment, we ask the question whether the frequency of adenomas in a neighbouring segment on one or both sides is larger among individuals with one or more adenomas in the index segment than in individuals with no adenomas in the index segment, relative to the number of adenomas in the pooled segments further apart. The method is based on applying the one sided Fisher-Irwin test to appropriate $2 \times 2$ tables. ${ }^{5}$

Figure 4 shows a general tendency of adenomas to occur relatively more frequently in segment(s) next to a colonic index segment than in segments further apart when the index segment is non-empty. The tendency of clustering of adenomas is only seen in segments next to the index segment. No difference in relative frequency of adenomas was found between segments remote from the neighbouring segments. The results for the rectum do not fall into the pattern found in the colon segments as seen in Figure 4. No obvious clustering of adenomas is shown in the sigmoid colon when the rectum is the index segment.

Although there is a statistically significant local clustering within individuals in our material, this effect is not particularly strong. The strength of the clustering effect may be roughly estimated from Figure 2 in women above 65 years of age. The probability that two adenomas randomly chosen from the pooled set are close (less than 0.3 apart) is about 1.7 times as high when the adenomas come from the same individual as when they come from different individuals.

\section{Discussion}

It has previously been pointed out that there is a shift of predilection site of adenomas from the left side of the large intestine at younger ages to the right side at older ages. ${ }^{16}$ This indicates an age related site specificity of adenomas in the large intestine in both young and older patients on population basis.

The present study shows that multiple adenomas in the individual patient have a tendency to occur closer together than expected from the general distribution of adenomas of the large intestine within each broad age group. We have also excluded that this effect of individual clustering can solely be ascribed to the age effect on the population basis 

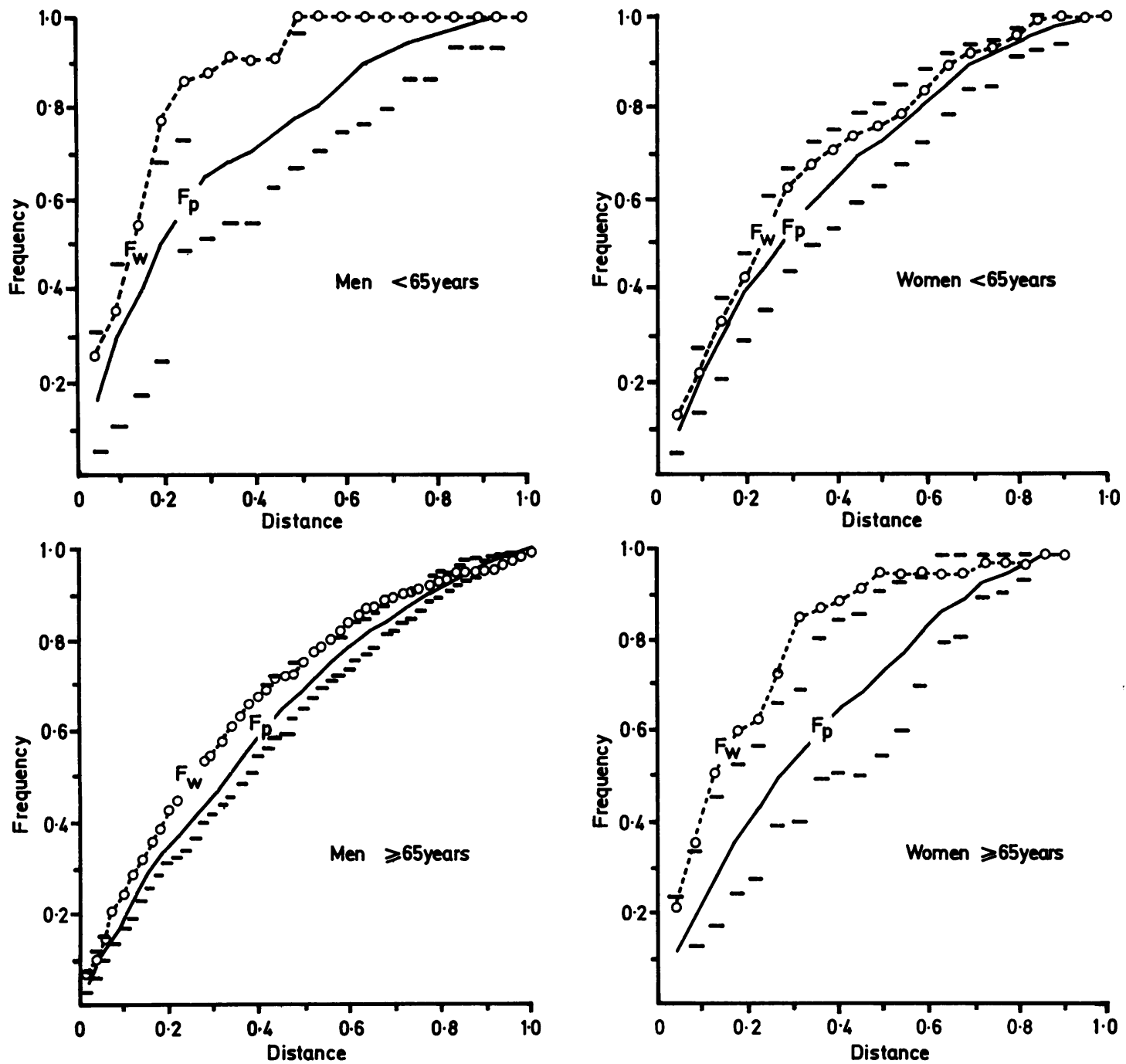

Fig. 2 Cumulative distribution functions for interadenoma distances within individuals, $F_{w}$, and in the pooled sample, $F_{p}$, for men and women above and below the age of $65 . F_{w}$ is the weighted average of the interadenoma distance distribution functions within separate individuals of age and sex group, the weights being as in one way analysis of variance. Dashed lines indicate a $95 \%$ tolerance limit for $F_{w}$, obtained by randomly allocating adenomas to individuals and for each allocation computing $F_{w}$.

mentioned above. The general effect of individual clustering is statistically significant, but not very strong and apparently clustering does not occur in all individuals with multiple adenomas. It should be borne in mind that adenomas may regress and disappear and the growth of additional adenomas probably occur at various times. ${ }^{78}$ The rather weak effect of clustering may, therefore, to some extent, be caused by regression of neighbouring adenomas or to a delay in the development of multiple adenomas around a single adenoma in some patients. Clustering often occurs in multiple areas of the large intestine, rather than being confirmed to one. A group of two or more adenomas in one part of the bowel does not therefore exclude the probability for adenomas to occur in other parts of the bowel. Clustering of multiple adenomas may occur around any of the colonic segments but is less 

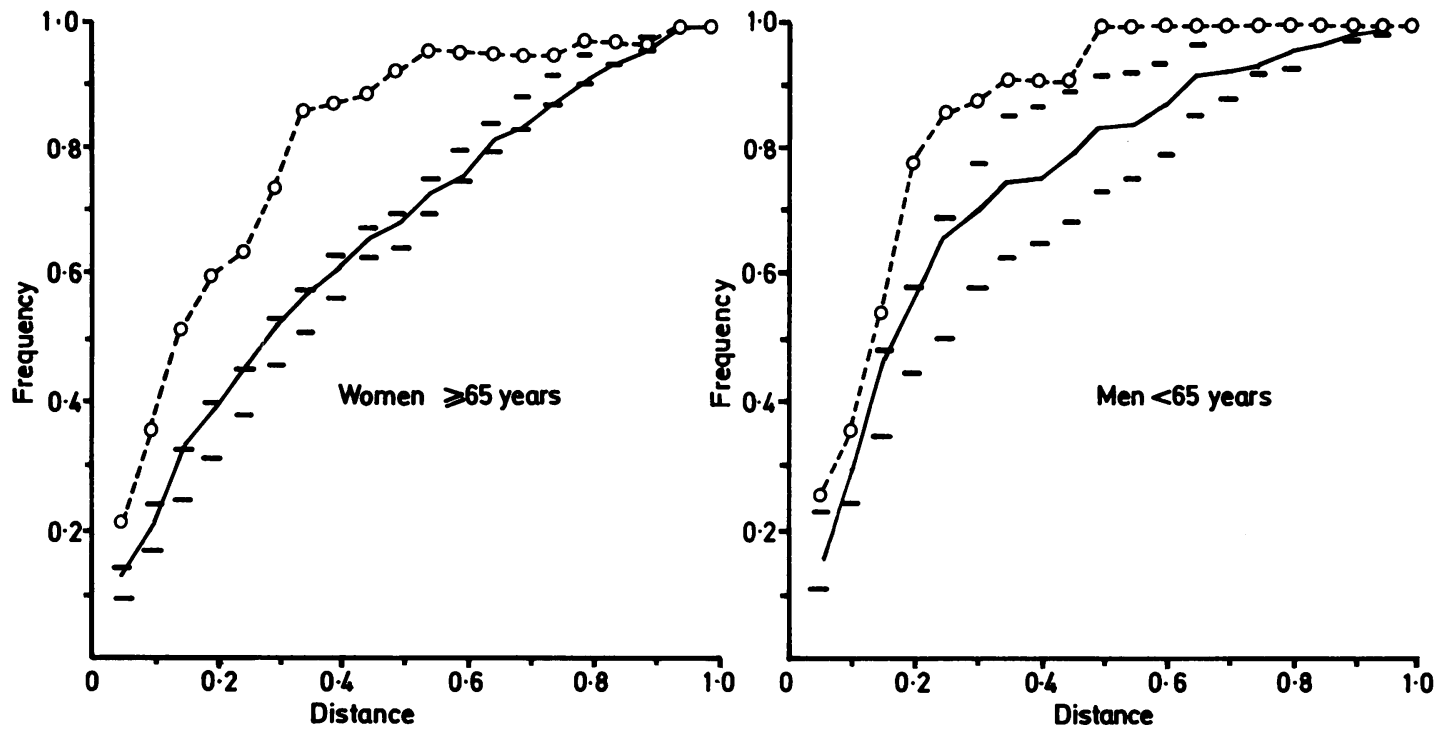

Fig. 3 Cumulative distribution functions for interadenoma distances within individuals, $F_{w}$, and within five year age groups, $F_{5}$, for women above the age of 65 and for men below that age. Dashed lines indicate a $95 \%$ tolerance limit for $F_{5}$ obtained by randomly allocating adenomas to age groups.

obvious in relation to adenomas of the rectum.

In this study we have looked upon clustering as a tendency of adenomas to occur close together on a line relative to the caecal pole and the lower end of rectum. We have not taken into consideration that adenomas with the same relative position between caecum and rectum may have different locations on the inner circumference of the bowel. This type of clustering would have required methods other than those we have used and seem to us to have a negligible effect on our conclusions.

Clustering of multiple polyps has previously been examined by Blatt ${ }^{9}$ who in a necropsy study found multiple polyps confined to the same or adjacent anatomical segments in $43 \%$ of all cases with multiple polyps. A clustering of adenomas around carcinomas when both occurred together was shown by Berge et al. ${ }^{10}$ Similarly, studies of synchronous carcinomas of the large intestine have shown that they tend to occur closer together than would have been expected by chance. ${ }^{11} 12$ Thomas et al 13 found in a study of 65 patients, each having two synchronous carcinomas of the large intestine, that both tumours were located in the same anatomical segment in 36 patients. Lasser ${ }^{14}$ in a study of 62 patients with synchronous carcinomas of the colon and rectum found the distance separating the tumours to be less than $20 \mathrm{~cm}$ in $84 \%$ of the cases. Thus, clustering has been shown to occur among multiple adenomas, among multiple carcinomas and between carcinomas and adenomas. The observations support the concept of a close relationship between adenomas and carcinomas.

The cause of clustering of adenomas may be explained as a local response to the growth of a single adenoma. The area around an adenoma may change and be more susceptible for the growth of additional adenomas. It is known that the mucosa at the edge of a carcinoma may be hyperplastic but this type of hyperplasia differs from that seen in adenomas and adenomas rarely occur in this area, adjacent to a carcinoma. More reasonable is the suggestion that the existence of clustering may be taken to indicate that in each individual the propensity for neoplastic development varies along the intestine, and that this pattern of variable risk differs from one individual to the other.

Adenomas of the rectum do not seem to fall into the pattern of clustering shown by those of other segments. The frequency of synchronous adenomas in the adjacent sigmoid colon is rather lower than expected. This may indicate a greater difference between the rectum and colon than among colonic segments with regard to factors responsible for adenomatous growth.

The authors thank Mrs Ellen Duus for technical assistance and Mrs Dagny Madsen for secretarial 

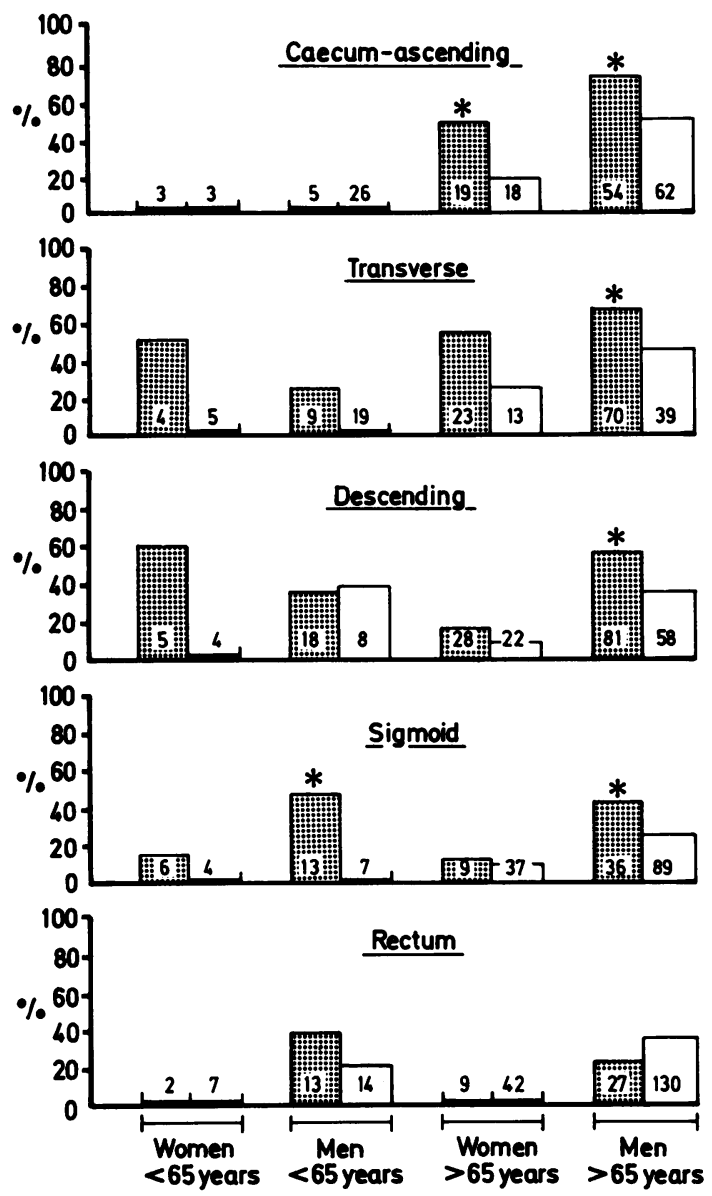

Fig. 4 Comparison of relative proportion of adenomas (in \%) occurring in segments next to (dark columns) and apart from (light columns) the index segment, which are coexisting with single or multiple adenomas in respective index segment (caecum-ascending, transverse, descending, sigmoid, rectum) in men and women above and below the age of 65. Number at the base of the columns is the total number of adenomas in each of compared groups of segments. * Symbolises that the difference between the compared groups of segments is statistically significant at the level of $5 \%$. assistance. This project was supported by the Norwegian Cancer Society.

\section{References}

1 Eide TJ, Stalsberg $\mathrm{H}$. Polyps of the large intestine in Northern Norway. Cancer 1978; 42: 2839-48.

2 Morson BC, Sobin LH. Histological typing of intestinal tumours. Geneva: WHO 1976.

3 Noether GE. Introduction to statistics. A nonparametric approach. Boston: Houghton Mifflin Co, 1976.

4 Schweder T. Investigating group specific clustering in univariate spatial data with many small groups. Biometrics 1984. (In press).

5 Hodges JL Jr, Lehmann EL. Basic concepts of probability and statistics. San Francisco: Holden-Day, 1964.

6 Vatn MH, Stalsberg H. Polyps of the large intestine in Oslo. A prospective autopsy study. Cancer 1982; 49: 819-25.

7 Cole JW, McKalen A, Powell J. The role of ileal contents in the spontaneous regression of rectal adenomas. Dis Colon Rectum 1961; 4: 413-8.

8 Knoernschild HE. Growth rate and malignant potential of colonic polyps: early results. Surg Forum 1963; 14: 137-8.

9 Blatt LJ. Polyps of the colon and rectum. Incidence and distribution. Dis Colon Rectum 1961; 4: 277-82.

10 Berge T, Ekelund G, Mellner G, Pihl B, Wenckert A. Carcinoma of the colon and rectum in a defined population. An epidemiological, clinical and postmortem investigation of colorectal carcinoma and coexisting benign polyps in Malmø, Sweden. Acta Chir Scand (suppl) 1973; 438.

11 Moertel CG, Bargen JA, Dockerty M. Multiple carcinomas of the large intestine. A review of the literature and a study of 261 cases. Gastroenterology 1958; 34: 85-98.

12 Ekelund GR, Pihl B. Multiple carcinomas of the colon and rectum. Cancer 1974; 33: 1630-4.

13 Thomas JF, Dockerty M, Waugh J. Multiple primary carcinomas of the large intestine. Cancer 1948; 1: 564-73.

14 Lasser A. Synchronous primary adenocarcinomas of the colon and rectum. Dis Colon Rectum 1978; 21: 20-2. 